\title{
Wafer Bifurcation as a Spontaneous Symmetry Breaking
}

\author{
Authors \\ Vincenzo Vinciguerra*, Antonio Landi, Giuseppe Luigi Malgioglio \\ STMicroelectronics, ADG R\&D \\ Stradale Primosole 50, 95121 Catania (Italy) \\ *Corresponding author: vincenzo.vinciguerra@st.com
}

\begin{abstract}
A connection between the phenomenon of wafer bifurcation and that of spontaneous symmetry breaking (SSB) has been established. Indeed, by developing an analytical approximation of the elastic energy of a wafer coated with a thin layer (e.g. a metal), it is shown as the elastic potential energy, interpreted as a quantity contributing to the thermodynamic free energy, can be investigated within the framework of the Landau theory of the second order phase transitions. The elastic energy of a bifurcated wafer is a complex function of the stress and the curvatures in the two perpendicular directions. In this work, it is shown as a translation of the coordinate system of the curvatures allows to gain a potential which has a "Mexican hat" shape. This is a distinctive trait of the phenomenon of spontaneous symmetry breaking (SSB). Moreover, it is shown as the values of the coordinates at the minimum of the SSB potential agrees with those provided by the theory. Bifurcation is hence a phenomenon that can also be interpreted as a spontaneous symmetry breaking where the rotational symmetry of a disc shaped wafer is broken. It occurs because of a lowering of the wafer energy in the SSB broken symmetry configuration. Keywords: Bifurcation, Wafer, Spontaneous Symmetry Breaking, Finite Element Analysis (FEA), Simulations.
\end{abstract}

\section{Introduction}

Wafer bifurcation [1] [2] [3] [4] [5] [6] occurs when the mismatch strain overcomes a specific and system dependent critical value. The result is an asymmetric warpage increase [7] [8]. For example, in a semiconductor wafer (considered as a symmetric disc) it determines a transition from a high symmetric configuration, namely a symmetric paraboloid (usually referred as the spherical case) to a lower symmetry, specifically an elliptic paraboloid (usually referred as the cylindrical case). In the work [9] we have investigated the phenomenon of bifurcation. In particular, an analytical model of the dependence of the bifurcation energy of the substrate on the residual stress has been developed for a metalized wafer and validated by exploiting Finite Element Analysis (FEA) methods. Some results are hereafter summarized. In Fig. 1, the normalized curvature versus normalized mismatch strain of a wafer, including the linear and nonlinear (large deformation) regimes and the case of the bifurcation regime [2]. Below the bifurcation point, the behavior is approximated by the Freund's equation [8] [10], whereas the behavior determined by the Stoney's theory is described by the line of slope 1 . In the bifurcation regime, the average curvatures of a bifurcated wafer lie on the line from the origin to the bifurcation point. In Fig. 2 the distribution of the directional deformation, along $\mathrm{z}$ in an $8 "$ silicon wafer, $500 \mu \mathrm{m}$ thick metalized with a $4.5 \mu \mathrm{m}$ Al layer, simulated with ANSYS 2021 $\mathrm{R} 1$ [11], is reported from ref [9].

In this work we want to evidence a link that exists between the elastic energy of a wafer and the phenomenon of the spontaneous symmetry breaking (SSB). SSB [12] is a mechanism which manifests in several fields of physics, ranging from second order phase transitions to particle physics mass diversification. It occurs when a configuration is energetically favored at the expenses of a loss of symmetry of the whole system. 


\section{SSB mechanism in the elastic energy of a bifurcated wafer}

The configuration that a metalized wafer assumes, at thermal equilibrium, is related to the minimum of the elastic potential energy $V$ accumulated in the substrate. This energy, which solves the role of a thermodynamic free energy, depends on the residual stress $\sigma$ and the curvature $\kappa$. As reported in [9], by reasoning on the linear regime, the non-linear approximation in the spherical case, and the extension to the bifurcation case, we can write the elastic energy of a bifurcated wafer as:

$$
V\left(\sigma, \kappa, \kappa_{\perp}\right)=-\frac{4}{3} h_{f} h_{s} \pi R^{2}\left[\sigma \frac{\kappa+\kappa_{\perp}}{2}-\frac{1}{24} \frac{E_{S}}{1-v_{S}^{2}} \frac{h_{S}^{2}}{h_{f}}\left(\kappa^{2}+2 v_{s} \kappa \kappa_{\perp}+\kappa_{\perp}^{2}\right)-\frac{E_{s}}{24} \frac{1}{h_{f}} \frac{R^{4}}{16} \kappa^{2} \kappa_{\perp}^{2}\right](1),
$$

where $\kappa$ and $\kappa_{\perp}$ are the principal curvatures of the bifurcated wafer, $h_{s}$ the substrate and $h_{f}$ the film thicknesses, $R$ the radius of the wafer, $E_{s}$ the Young's module and the $v_{s}$ Poisson's coefficient of the substrate. This equation can be modified further. Indeed, by rescaling the curvatures as $K=\frac{\kappa}{\kappa_{b i f}}$ and $K_{\perp}=\frac{\kappa_{\perp}}{\kappa_{b i f}}$ with respect to the curvature $\kappa_{b i f}$, which marks the onset of the bifurcation, and by defining a new energy scale as $F_{0}=-\frac{4}{3} h_{f} h_{s} \pi R^{2} \frac{1}{24} \frac{E_{s}}{1-v_{s}^{2}} \frac{h_{s}^{2}}{h_{f}} \kappa_{b i f}^{2}$, as well by writing a new factor $\mathcal{H}$ as $\mathcal{H}=\frac{24\left(1-v_{S}^{2}\right)}{E_{S}} \frac{h_{f}}{h_{S}^{2}} \frac{1}{\kappa_{b i f}}$, the elastic energy of a bifurcated wafer can be simplified as:

$$
V\left(\sigma, K, K_{\perp}\right)=F_{0}\left[\sigma \mathcal{H} \frac{K+K_{\perp}}{2}-\left(K^{2}+2 v_{S} K K_{\perp}+K_{\perp}^{2}\right)-\left(1-v_{s}\right) K^{2} K_{\perp}^{2}\right](2)
$$

In fig.3 the 3D plot of $V\left(\sigma, K, K_{\perp}\right) / F_{0}$ as a function of $K$ and $K_{\perp}$, rescaled by a factor of 0.15 , has been reported for the case of $\sigma \mathcal{H}=25, v_{s}=0.27$. The green humped curve is determined by the intersection of the surface with the plane of equation $K+K_{\perp}=5.2$.

Now, to make evident the mechanism of SSB, we can consider, in eq. 2, a further change of variables, namely, the translation of the coordinates $K$ and $K_{\perp}$ :

$$
\left\{\begin{array}{l}
K^{\prime}=K-\frac{\sigma}{\sigma_{b i f}} \\
K_{\perp}^{\prime}=K_{\perp}-\frac{\sigma}{\sigma_{b i f}}
\end{array}\right.
$$

and the evaluation of the elastic energy along the plane of equation $\frac{K+K_{\perp}}{2}=\frac{\sigma}{\sigma_{b i f}}$.

In this case, being $K_{\perp}^{\prime}=-K^{\prime}$, eq. 2 becomes:

$V\left(\sigma, K^{\prime}\right)=F_{0}\left[\sigma \mathcal{H} \frac{\sigma}{\sigma_{\text {bif }}}-2\left(1+v_{s}\right) \frac{\sigma^{2}}{\sigma_{\text {bif }}^{2}}-2\left(1-v_{s}\right) K^{\prime 2}-\left(1-v_{s}\right)\left(-K^{\prime 2}+\frac{\sigma^{2}}{\sigma_{b i f}^{2}}\right)^{2}\right](4)$

By focusing on the terms depending on $K^{\prime}$, eq. 4 can be rescaled in a dimensionless potential $F$ :

$F=\frac{1}{1-v_{s}}\left[\sigma \mathcal{H} \frac{\sigma}{\sigma_{b i f}}-2\left(1+v_{s}\right) \frac{\sigma^{2}}{\sigma_{b i f}^{2}}\right]-\frac{V\left(\sigma, K^{\prime}\right)}{F_{0}\left(1-v_{s}\right)}-\frac{\sigma^{4}}{\sigma_{b i f}^{4}}=2 K^{\prime 2}+\left(-K^{\prime 2}+\frac{\sigma^{2}}{\sigma_{b i f}^{2}}\right)^{2}-\frac{\sigma^{4}}{\sigma_{b i f}^{4}}(5)$

This potential grasps in its essence the physics of the SSB, which occurs during the bifurcation of a wafer. Indeed, the potential $F$ depends on the even power of $K^{\prime}$, namely $K^{\prime 2}$ and $K^{\prime 4}$. It has two minima of equal energy, symmetrical positioned at $K^{\prime}= \pm \sqrt{\sigma^{2} / \sigma_{b i f}^{2}-1}$, which correspond to the principal curvatures of the bifurcated wafer. 
In Fig.4, we report the plot of the potential $F$ as a function of $K^{\prime}$ for several values of $\frac{\sigma}{\sigma_{b i f}} \geq 1$. It describes the mechanism of the SSB. Indeed, it is evident how the potential $F$ is consistent with the description of a "Mexican-hat" shaped potential, usually invoked in the description of the SSB phenomenon and the thermodynamical second order phase transitions [12]. Moreover, the minima of the potential have values which go lower and lower in energy and step away from the origin as $\frac{\sigma}{\sigma_{b i f}}$ increases.

\begin{tabular}{|l|l|l|} 
Fig. 1. Normalized curvature versus normalized \\
mismatch strain of a wafer, including the linear \\
and nonlinear (large deformation) regimes and the \\
case of the bifurcation regime. Below the \\
bifurcation point, the behavior is approximated by \\
the Freund's equation $[10]$, whereas the behavior \\
determined by Stoney's theory is described by the \\
line of slope 1. In the bifurcation regime, the \\
average curvatures of a bifurcated wafer lie on the \\
line from the origin to the bifurcation point.
\end{tabular}

\section{Conclusion}

In conclusion, we demonstrated as wafer bifurcation can be described in terms of a spontaneous symmetry breaking mechanism, which involves the elastic energy of the wafer. The SSB mechanism occurs because of a lowering of the energy of the bifurcated wafer, but at the 
expenses of a loss of symmetry of the system, namely the cylindric symmetry. These findings may result in a further understanding and control of the phenomenon of bifurcation.

\section{References}

[1] C. Zhu, "Experimental and theoretical investigation of bifurcated wafer warpage evolution in the wafer level packaging processes," Journal of Materials Science: Materials in Electronics, vol. 31, no. 19, p. 16531-16538, 2020.

[2] V. Vinciguerra and A. Landi, "On the Way to understand the Warpage in 8' Taiko Semiconductor Wafers for Power Electronics Applications (Si and SiC)," in 2021 22nd International Conference on Thermal, Mechanical and Multi-Physics Simulation and Experiments in Microelectronics and Microsystems (EuroSimE), Virtual, 2021.

[3] H. Liu, J. Han, X. Tian, F. Dong, S. Chen and L. Lu, "Accurate determination of bifurcation points for ground silicon wafers considering anisotropy using FEM method," Materials Research Express, vol. 6, no. 9, 72019.

[4] D. K. Shin and J. J. Lee, "Analysis of asymmetric warpage of thin wafers on flat plate considering bifurcation and gravitational force," IEEE Transactions on Components, Packaging and Manufacturing Technology, vol. 4, no. 2, pp. 248 - 258, Feb. 2014.

[5] N. J. Salamon and C. B. Masters, "Bifurcation in isotropic thinfilm/substrate plates," International Journal of Solids and Structures, vol. 32, no. 3-4, pp. 473-481, Febbruary 1995.

[6] C. B. Masters and N. J. Salamon, "Geometrically nonlinear stress-deflection relations for thin film/substrate systems with a finite element comparison," J. Appl. Mech. , vol. 61, no. 4, pp. $872-878$ (7 pages), Dec 1994.

[7] L.B. Freund and S. Suresh, Thin Film Materials: Stress, Defect Formation and Surface Evolution, Cambridge: Cambridge University Press, 2004.

[8] L. B. Freund, "Substrate curvature due to thin film mismatch strain in the nonlinear deformation range," Journal of the Mechanics and Physics of Solids, vol. 48, no. 6, pp. 1159-1174, 2000.

[9] V. Vinciguerra, G. L. Malgioglio and A. Landi, "Modelling the Elastic Energy of a Bifurcated Wafer: A benchmark of the Analytical Solutions vs. the ANSYS Finite Element Analysis," Composite Structures, 114996, vol. 281, 2022.

[10] L. B. Freund, J. A. Flora and E. Chason, "Extensions of the Stoney formula for substrate curvature to configurations with thin substrates or large deformations," Applied Physics Letters, vol. 74, no. 14, p. 1987, 1999.

[11] ANSYS Mechanical Enterprise interface Workbench release 2021 r1.

[12] A. Beekman, L. Rademaker and J. van Wezel, "An introduction to spontaneous symmetry breaking," SciPost Physics Lecture Notes, 2019.

[13] "http://www.geogebra.org," [Online]. 